\begin{tabular}{|c|c|c|c|c|c|c|}
\hline \multicolumn{7}{|c|}{ Baryumhydroxyd: } \\
\hline \multicolumn{2}{|c|}{$\begin{array}{l}\text { Spannun } \\
\text { gefunden }\end{array}$} & \multirow{2}{*}{$\begin{array}{l}\text { in Volt } \\
\text { berechnet } \\
\text { aus Nr. } 8 \\
\text { I,9.29 }\end{array}$} & \multirow{2}{*}{$\begin{array}{l}\text { Differenz } \\
+0,0015\end{array}$} & \multirow{2}{*}{$\begin{array}{c}c \\
\text { gefunden } \\
0,6\end{array}$} & $\begin{array}{c}C \\
\text { berechnet } \\
\text { aus } \mathrm{Nr} .8\end{array}$ & Differ \\
\hline 1. & $\mathrm{I}, 9 \mathrm{II} 4$ & & & & 0,53 & $-0,07$ \\
\hline 2. & 1,9225 & $I, 924^{8}$ & $-0,0007$ & 1,6 & 1.7 & $+0,1$ \\
\hline 3. & 1,9334 & $\mathrm{r}, 9343$ & $+0,0009$ & 3,5 & 3,2 & $-0,3$ \\
\hline 4. & $1,935^{\circ}$ & $I, 9365$ & $+0,0015$ & 4,2 & 3,7 & $-0,5$ \\
\hline 5 . & $\mathrm{I}, 9400$ & 1,9399 & $-0,0001$ & 5.5 & 5,7 & $+0,2$ \\
\hline 6. & $I, 947^{\circ}$ & 1,9483 & $+0,0013$ & II,$\infty$ & 9,9 & $-I, I$ \\
\hline 7 . & $1,953^{\circ}$ & $1,95^{21}$ & $-0,0009$ & $15, \infty$ & 16,2 & $+\mathrm{r}, 2$ \\
\hline 8. & 1,9574 & {$[\mathrm{I}, 9574]$} & - & 23,3 & {$[23,3]$} & - \\
\hline 9. & $\mathrm{I}, 96 \mathrm{II}$ & I,96 I3 & $+0,0002$ & $32, \infty$ & 30,9 & $-1,1$ \\
\hline & 1,9628 & $1,96 r_{4}$ & $-0,0012$ & $33, \infty$ & 35,7 & $+2,7$ \\
\hline 1. & $x, 9618$ & $\mathrm{I}, 9645$ & $+0,0027$ & 42,00 & 33,4 & $-8,6$ \\
\hline
\end{tabular}

Natriumhydroxyd:

\begin{tabular}{|c|c|c|c|c|c|c|}
\hline \multirow[b]{2}{*}{ I, } & \multicolumn{3}{|c|}{$\begin{array}{l}\text { berechnet } \\
\text { aus } \mathrm{Nr}, 5\end{array}$} & \multicolumn{3}{|c|}{$\begin{array}{l}\text { berechnet } \\
\text { aus Nr. } 5\end{array}$} \\
\hline & $\mathrm{I}, 7925$ & I, 7944 & $+0,0019$ & ז5,7 & $\mathbf{I}_{5}, 00$ & $-0,7$ \\
\hline 2. & $\mathbf{1}, 8 \mathbf{I}_{45}$ & $\mathrm{I}, 8 \mathrm{I}_{4}$ & $+0,0004$ & 37,7 & $37, \infty 0$ & $-0,7$ \\
\hline & 1,8135 & $\mathbf{I}, 8 \mathrm{I}^{\mathrm{I}}$ & $+0,0016$ & $3^{8,00}$ & 35 & $-2,5$ \\
\hline & 1,8246 & $I, 8240$ & $-0,0006$ & 54,8 & $56, I$ & +1.3 \\
\hline & $\mathrm{r}, 8300$ & {$\left[1,83^{\circ}\right]$} & - & $70, \infty 0$ & {$[70,-\cdots]$} & - \\
\hline & I, 8328 & I,8325 & $-0,0003$ & 77,7 & 78,5 & $+0,8$ \\
\hline & I, $833^{2}$ & $\mathrm{I}, 8342$ & $+0, \infty 010$ & 83,3 & 79,8 & $-3,5$ \\
\hline
\end{tabular}

Die Uebereinstimmung zwischen Beobachtung und Rechnung ist als eine sehr gute zu bezeichnen. Die auftretenden Differenzen sind auf Versuchsfehler zurückzuführen, namentlich, da sie bald positiv, bald negativ erscheinen. In der Tabelle des Baryumhydroxydes ist die Differenz bei Nr. I I viel grösser wie bei den andern Werten. Es rührt dies daher, dass die Grenze der Löslichkeit des Baryums in Quecksilber bei Io $^{0}$ uberschritten ist und sich demgemäss zwar eine grössere Konzentration ergiebt, aber keine höhere Spannung wie bei Nr. Io, welch letztere also den Wert für die gesăttigte Lösung darstellt. Die $33 \mathrm{ccm} 1 / 10 \mathrm{n} . \mathrm{HCl}$ entsprechen circa $0,23 \%$ Baryum, eine Zahl, welche mit der von Dr. Kerp gefundenen und mir gütigst mitgeteilten, circa $0,24^{0}$, übereinstimmt.

Aus der Thatsache, dass die experimentell ermittelten Werte der Spannungen für das Baryumamalgam mit den berechneten ubereinstimmen, folgt, dass Baryum in Quecksilber als Atom gelöst ist.

\title{
ÜBER EINIGE FORMEN DER GEBRÄUCHLICHSTEN GALVANISCHEN ELEMENTE.
}

\section{Von Emil Petersen.}

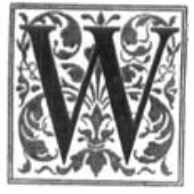

iewohl die galvanischen Elemente als Stromquellen, wo es sich um etwas bedeutendere Arbeitsleistung handelt, immer mehr und mehr verdrăngt werden, ist man doch noch an vielen Stellen und in besonderen Fällen zu dieser Quelle verwiesen. Bei der Vorbereitung einiger elektrochemischer Untersuchungen war ich in dieser Lage und hatte Gelegenheit, einige Beobachtungen, die gebräuchlichsten Elemente betreffend und einige Abänderungen derselben zu machen, die vielleicht auch anderen nützlich sein können.

Bekanntlich sind im Laufe der Zeit viele Variationen der gebräuchlichen Elemente vorgeschlagen und geprüft worden. Während man aber hier früher zum Teil auf eine empirische Zufälligkeit angewiesen war, bietet sich nun in der von Nernst ${ }^{1}$ ) entwickelten osmotischen Stromtheorie ein leitendes Prinzip dar, welches

I) Zeitschrift für physikalische Chemie 4, $129 \mathrm{ff}$. (1889). wohl auch auf dieses Gebiet anwendbar ist. Wohl lassen sich die Gleichungen, die für die reversibeln Ketten entwickelt worden sind, hier nicht direkt benutzen; doch müssen die allgemeinen Prinzipien der Theorie für die Ursache der Stromerzeugung auch hier Geltung haben.

Ich habe für die verschiedenen, unten zu erwähnenden Kombinationen die elektromotorische Kraft mittels kleiner Elemente mit möglichst reinem Stoffe genau gemessen. Die Methode war die von Ostwald ${ }^{1}$ ) beschriebene, mit dem Lippmannschen Kapillarelektrometer. Bei der praktischen Prüfung von grösseren Elementen ist zur Messung der elektromotorischen Kraft während des Stromschlusses ein Voltmeter nach System Desprez-d'A rsonval benutzt. Dasselbe war in $1 / 10$ Volt geteilt und mit einem geaichten Präzisionsinstrumente verglichen.

Man kann wohl die gebräuchlichsten Arbeitselemente in zwei Hauptgruppen teilen: I. „Bunsen-Elementc" (in erweitertem Sinne), in

I) Ibid. 5, 47 I (I89o), 
welchen die Kathode unangreifbar und von einem "depolarisierenden" Oxydationsmittel umgeben ist; und 2. "Daniell-Elemente", deren Wirksamkeit auf Losung eines mehr und Ausfallung eines weniger positiven Metalles beruht. Vom Typus des „Bunsen-Elementes" sind viele Variationen gepraft worden, doch stehen sie bekanntlich alle in Konstanz und Ausgiebigkeit hinter der ursprünglichen Form: Zink/verdannte Schwefelsäure - konzentrierte Salpetersäure | Kohle zuruck. Statt der Schwefelsäure in dieser kann man, um die lästige und meistens nicht hinreichende Amalgamierung der Zinkelektrode zu vermeiden, auch eine beliebige neutrale Salzlösung benutzen. Dadurch wird nur der innere Widerstand des Elementes geändert, während die elektromotorische Kraft unverändert bleibt, weil der Losungsdruck des Zinkes und damit die Potentialdifferenz einer Flussigkeit gegenuber, die keine Zinkionen vorher enthalt, dieselben bleiben. Für Zink-Chlornatriumlossung in Kombination mit einer Kalomel-Normalelektrode fand ich in fanf Versuchen, in welchen die Chlornatriumlösung vom Verhaltnis $I: 3$ bis I : 5 variierte, was das Resultat nur wenig beeinflusst:

$$
\begin{aligned}
& \text { I, I62 Volt, } \\
& \text { I, I58 " } \\
& \text { I, I59" } \\
& \text { I, I } 48 " \\
& \text { I,I63 " }
\end{aligned}
$$

Daraus ergiebt sich durchschnittlich für die Potentialdifferenz $\mathrm{Zn} \mid \mathrm{NaCl}$-Losung: 0,598 Volt der Theorie gemäss etwas grösser als einer normalen Zinksulfatlösung gegenuber, für welche Neumann') $\mathbf{0 , 5 2 4}$ Volt fand. Mit denselben Apparaten, wie oben angewendet, fand ich fur die Zinksulfatlossung 0,525 Volt.

Ersetzt man die Schwefelsaure im BunsenElemente mit einer konzentrierten Kochsalzlösung, so wird auch der innere Widerstand eben so klein wie mit Schwefelsäure; eine Lősung von Chili-Salpeter (Verhältnis $4: 5$ ) macht ihn ein wenig grosser.

Viel schwieriger ist ein Ersatz fur die Salpetersăure zu finden, um die mit dieser verbundene Entwicklung von abelriechenden und die Metallkontakte angreifenden Dämpfen zu vermeiden. Wohl bleibt die elektromotorische

I) Zeitschr. phys. Chem. 14, S. 229.
Kraft mit anderen starken Oxydationsmitteln fast dieselbe oder wird noch höher; so fand ich fur die folgenden Oxydationsmittel in Kombination mit der Zink-Chlornatriumelektrode (indem far jede Messung eine frische Kohlenelektrode benutzt wurde):

Mit Kaliumpermanganat (Verhaltnis I:16) . 1,678 Volt, mit Wasserstoffhyperoxyd (ca. einprozentiges, mit I Vol. verdunnter Schwefelsăure vermischt) . . . . . . . . I,699 " mit Bleihyperoxyd (in verdünnter

Schwefelsäure suspendiert) . . I,99 I " mit Chlornatron (ohne uberschüssi-

ges Chlor) 2,021

Aber alle diese Kombinationen stehen in Konstanz und Ausgiebigkeit nicht nur hinter dem Salpetersăure-Element, sondern auch hinter dem Chromsăure-Element zurück.

Die Ursachen dazu düften wesentlich zwei sein: erstens ist die "Depolarisation" unvollständig, d. h. neben der Hauptreaktion findet Wasserstoffentwicklung bei der Kathode statt; zweitens bleiben (wie auch beim ChromsäureElement) die Reaktionsprodukte in der Lossung, was zunehmend die Potentialdifferenz bei der Kathode herabsetzt. Dass dieses letztere beim Salpetersăure-Element nicht der Fall ist, muss als eine wesentliche Bedingung der vorzaglichen Konstanz dieses Elementes angenommen werden.

Um zu versuchen, die erstgenannte Ursache zu heben, ist es naheliegend, als Kathodenflüssigkeit eine Lősung zu wăhlen, die kein Sauerstoffsalz enthält, sondern entweder ein solches, wo das Kation făhig ist, niedrigere Valenz anzunehmen, unter entsprechender $\mathrm{Ab}$ gabe positiver Elektrizităt, oder umgekehrt ein solches, dessen Anion höhere Valenz annehmen und negative Elektrizităt aufnehmen kann. Ich habe ein Element mit einem Salze beider Arten năher gepruft.

Wenn Eisenchlorid durch Zink zu Chlorar reduziert wird:

$$
\mathrm{Zn}+2 \mathrm{FeCl}_{3}=\mathrm{ZnCl}_{2}+2 \mathrm{FeCl}_{2},
$$

so geschieht dies, der Theoric gemäss, dadurch, dass ein positiv geladenes Zinkion in Lösung geht, während gleichzeitig zwei Ferriionen in Ferroionen abergehen, unter Abgabe derselben 
Menge positiver Elektrizität, und dieses scheint beim ersten Anblick keine Wasserstoffentwicklung veranlassen zu können. Die obenstehende Reaktion entspricht einer Wärmeentwicklung von $573 \mathrm{~K} .{ }^{1}$.

Die Kombination: $\mathrm{Zn} \mid \mathrm{NaCl}$-Lösung $\mathrm{FeCl}_{3}$-Lösung ! Kohle ist schon von Buffa) I854 auf Veranlassung Liebigs untersucht worden. Ich habe - zum Teil ehe ich auf die Abhandlung von Buff aufmerksam war - das Element näher geprüft, und da meine Resultate etwas von den Angaben Buffs abweichen und ich auch die Untersuchung mehr erschöpfend durchgeführt habe, teile ich hier in aller Kürze die Hauptresultate mit

Die elektromotorische Kraft wurde teils direkt bestimmt, teils, indem ich die einzelnen Potentialdifferenzen bei den Elektroden maass. Gcfunden wurde:

$$
\begin{aligned}
& \mathrm{Zn} \mid \mathrm{NaCl} \text {-Lösung }-\mathrm{Fe} \mathrm{Cl}_{3} \text { 6aq-Lösung } \mid \text { Kohle } \\
& \begin{array}{c}
(\mathrm{I}: 5) \\
0,598 \text { Volt } \underset{\text { Gefunden } \underset{\mathrm{I}, 720 \text { Volt, }}{\longrightarrow}}{\longrightarrow} \text { I, I 29 Volt }
\end{array} \\
& \text { berechnet 1,727 }
\end{aligned}
$$

Ich habe Elemente mittlerer Grösse mit dieser Kombination vielfach $z u$ verschiedenen $Z$ wecken benutzt. Sie sind nicht konstant, aber übertreffen doch in dieser Beziehung $z$. B. das Leclanché-Element; wie dieses können sie beliebig lange ohne Stromentnahme stehen und sind dazu leichter zusammenzustellen. In der Praxis empfiehlt es sich, eine konzentrierte Eisenchlorid-Lösung (4:I) zu benutzen und diese mit etwa $1 / 4$ Vol. Chlornatriumlösung ( $1: 4)$ zu vermischen. Solche Elemente können bei mässiger Stromentnahme - nicht aber I Amp. - stundenlang konstant bleiben; bei weiterem Stromschluss sinkt die elektromotorische Kraft allmählich, steigt aber (wie beim LeclanchéElement) wieder, wenn das Element ohne Stromschluss steht. Der innere Widerstand eines Elementes mittlerer Grösse ist o,3 bis o,4 Ohm. Bei starkerer Stromentnahme, bezw. Kurzschluss, bemerkt man eine deutliche Gasentwicklung bei der Kohlenelektrode, und in allen Fällen scheidet sich beim Gebrauche reichlich Eisenoxydhydrat

I) J. Thomsen, Thermochem. Unters. III, S. 276 und 294 .

2) Liebigs Annalen XVI, S. II7. bei der Zinkanode ab. Dieses zeigt, dass der durch die obenstehende Gleichung ausgedrückte Prozess nur teilweise stattfindet, während zugleich wegen hydrolytischer Dissociation der folgende eintritt:

$$
\begin{gathered}
3 \mathrm{Zn}+2 \mathrm{FeCl}_{3}+6 \mathrm{H}_{2} \mathrm{O}={ }_{3} \mathrm{ZnCl}_{2} \\
+2 \mathrm{Fe}(\mathrm{OH})_{3}+3 \mathrm{H}_{2},
\end{gathered}
$$

dazu kommt noch, dass das gebildete Eisenchlorür die Potentialdifferenz bei der Kathode herabsetzt.

Ist beim Gebrauch des Elementes die ganze Chloridmenge zu Chlorür reduziert - was leicht durch Titrierung mit Kaliumpermanganat festzustellen ist -, zeigt es nach einiger Zeit eine elektromotorische Kraft von ca. 0,5 Volt, die bei weiterem Stromschlusse ziemlich lange konstant bleibt. Verfolgt man die Wirksamkeit des Elementes in diesen Phasen, so zeigt sich folgendes. Die elektromotorische Kraft der Kombination:

$$
\mathrm{Zn}\left|\begin{array}{cc}
\mathrm{NaCl} \text {. Lösung }-\mathrm{FeCl}_{2} \text {-Lösung } \\
(\mathbf{I}: 4)
\end{array}\right| \text { Kohle }
$$

wurde zu I,29o Volt gemessen, also bedeutend niedriger als mit dem Chlorid. Die Reaktion ist hier selbstverständlich:

$$
\mathrm{Zn}+\mathrm{FeCl}_{2}=\mathrm{ZnCl}+\mathrm{Fe},
$$

was einer Wärmeentwicklung von $\mathrm{I} 28,9 \mathrm{~K}$. entspricht ${ }^{1}$ ). - Steht ein solches Element einige Zeit geschlossen, so zeigt sich die Kohlenelektrode mit einer grauen, glänzenden Schicht von elektrolytisch gefälltem Eisen äberzogen: die Kohlenelektrode ist zu einer Eisenelektrode geworden. Die elektromotorische Kraft der Kombination:

$$
\mathrm{Zn}\left|\begin{array}{cc}
\mathrm{NaCl} \text {-Lösung }-\mathrm{Fe} C \mathrm{Cl}_{2} \text {-Lösung } \\
(\mathbf{1}: 4)
\end{array}\right| \mathrm{Fe}
$$

wurde zu o,537 Volt bestimmt; bei der Eisenelektrode geht hier der Potentialsprung, der durch Messung in Kombination mit der KalomelNormalelektrode zu $0,05^{\mathrm{r}}$ Volt gefunden wurde - vom Metall zur Lösung, also gegen den Strom des Elementes.

Das inkonstante und irreversible Element des Bunsen-Typus ist also hier zu einem konstanten und reversiblen Elemente cles DaniellTypus geworden. Ein Element mittlerer Grösse nach der letztgenannten Kombination zusammengestellt, mit einem schmiedeeisernen Hohlcylinder

I) Thermochem. Unters., Bd. III, S. 283 . 
als Kathode, der von gesättigter Eisenchlorürlösung (mit festen Krystallen ausgefüllt) umgeben war, zeigte beim Schluss durch das Ampèremeter dreimal 24 Stunden hindurch einen kon. stanten Strom von $\mathrm{I}, 20$ Ampère.

Ist beim Gebrauch des Eisenchlorid-Elementes diese Phase erreicht, muss man bei Erneuerung der Kathodenflüssigkeit zugleich das Eisen (mittels verdünnter Salzsäure) von der Kohle entfernen. Die Potentialdifferenz $\mathrm{FeCl}$-Lösung $\mid F e$, die von der Lösung zum Metall geht, wurde $z u \quad 0,273$ Volt bestimmt, ist also kaum ein Viertel der Potentialdifferenz zwischen Eisenchloridlosung und Kohle.

Lasst man im Eisenchlorid-Element den porösen Thoncylinder weg, indem man z. B. eine Zinkplatte und eine Kohlenstange, durch ein Holzstück getrennt, zusammenbindet und diese in die Eisenchloridlösung senkt, so giebt das Voltmeter eine Spannung von 1,60 Volt an; durch eine Batterie solcher Elemente, die sehr leicht aufzubauen ist, kann man sich für kürzere Zeit hochgespannte Ströme verschaffen.

Ersetzt man im Eisenchlorid-Elemente die Zinkelektrode durch eine Eisenelektrode, so wird voraussichtlich die elektromotorische Kraft des Elementes um ca. 1/2 Volt herabgesetzt, weil die Potentialdifferenz des Eisens dem Elektrolyt gegenüber um diese Grösse kleiner ist als die des Zinkes. Ich fand für

$$
\mathrm{Fe} \mid \begin{gathered}
\mathrm{NaCl} \text {-Lösung : 0,057 Volt. } \\
(\mathrm{I}: 4)
\end{gathered}
$$

Lässt man auch hier den porösen Thoncylinder weg, entsteht die einfache Kombination:

Eisen-Eisenchloridlösung-Kohle.

Sie zeigt die elektromotorische Kraft von o,9o Volt, die aber beim Stromschluss bald sinkt; mit einem Strome von ca. I Ampère war sie in 2 Stunden auf 0,70 Volt gesunken.

Um die kathodische Polarisation auszuschliessen, wurde versucht, statt Eisenchlorid Ferridcyankalium in der Kathodenlosung anzuwenden. Dieses Salz ist nur elektrolytisch dissociiert, und scin Anion hat Fähigkeit, höhere Valenz anzunehmen und grössere negative Ladung aufzunehmen. Es ist also Beispiel eines Salzes der zweiten (S. 262) genannten Art.

Der Prozess wird anfangs der folgende sein (beispielsweise mit Chlorkalium in der Anodenlösung):
$\mathrm{Zn}+2 \mathrm{KCl}+2 \mathrm{~K}_{3} \mathrm{FeCl}_{6}=\mathrm{ZnCl}_{2}+2 \mathrm{~K}_{4} \mathrm{Fe}_{e} \mathrm{Cy}_{6}$. Diesem entspricht cine Wärmeentwicklung von I I 7 I K. ${ }^{1}$ ).

Hier ist nun zu beachten, dass das gebildete Ferrocyankalium auf den Prozess im Elemente, der anfangs ohne Zweifel rein nach der obenstehenden Gleichung verläuft, späterhin störend einwirkt und durch seine Anwesenheit an und für sich die elektromotorische Kraft des Elementes herabsetzt. Dies geht aus den untenstehenden Messungen hervor. Ich bestimmte die elektromotorische Kraft kleiner Elemente mit Zink in Chlornatriumlösung $(\mathrm{I}: 4)$ und Kohle teils in reinen Lösungen von Ferridcyankalium oder von Ferrocyankalium, teils in Mischungen beider Lösungen in verschiedenen Verhaltnissen; gefunden wurde:

$$
\begin{gathered}
\text { Mit reinem Ferridcyankalium . I,438 Volt, } \\
\text { Ferridcyankalium und ganz } \\
\text { wenig Ferrocyankalium . I,40 " } \\
\text { 3/4 Vol Ferridcyankalium und } \\
\text { 1/4 Vol Ferrocyankalium. I,332 " } \\
\text { gleichem Vol der beiden } \\
\text { Lösungen . . . . . I,323 " } \\
1 / 4 \text { Vol Ferridcyankalium und } \\
3 / 4 \text { Vol Ferrocyankalium . I,292 " } \\
\text { reinem Ferrocyankalium. . I,I05 " }
\end{gathered}
$$

Beim Stromschluss von Elementen gewöhnlicher Grösse ist keine Spur von Gasentwicklung bei der Kathode zu entdecken, und thatsächlich ist auch die Konstanz relativ besser als die des Eisenchlorid-Elementes. Ströme, die nicht I Ampère überschreiten, können viele Stunden lang konstant bleiben. Setzt man den Stromschluss fort, bis fast alles Ferridcyankalium zu Ferrocyankalium reduziert ist, nimmt doch die elektromotorische Kraft bedeutend ab. In einer Batterie von sechs Elementen mittlerer Grösse, nacheinander geschaltet, verbrauchte ro $\mathrm{ccm}$ der Kathodenlösung, nachdem die Batterie ungefähr die berechnete Anzahl Ampèrestunden

1) J. Thomsea, Thermochem. Inters., Bd. III, S. 235 und 276 . - Berthelot, Ann. de chim. et de plyys. (5), T. 5, S. 464. - Joannis, Ibid. (5), T. 26, S. 5r3ff. - Mittels der bekannten Helmholtzschen Gleichung lässt sich berechnen - freilich unter der nicht ganz zutreffenden Voraussetzung, dass die Elemente reversibel sind --, dass das Ferridcyankalium-Element mit Wärmeentwicklung, das Eisenchlorid-Elenent unter Wärmeabsorption arbeitet. 
geleistet hatte, durch direkte Titrierung mit Kaliumpermanganat $I 8,0 \mathrm{ccm}$ und nach Reduktion mit Natriumamalgam $\mathbf{I} 8,4 \mathrm{ccm}$. Die elektromotorische Kraft der Batterie war anfangs 8,40 Volt, sank bald auf 8,00 Volt, wobei sie bei nicht allzu starker Stromentnahme stundenlang stehen blieb, und war schliesslich 5,40 Volt.

Der innere Widerstand ist für Elemente mittlerer Grösse nur ca. 1/4 Ohm. Die Kathodenflüssigkeit lăsst sich im Laboratorium durch Zuleitung von Chlor leicht erneuern, wodurch die Kosten beim Gebrauch des Elementes sehr herabgesetzt werden. Die Haltbarkeit beim Stehen ohne Stromschluss scheint sehr gross zu sein.

Vollkommen konstant sind bekanntlich nur die reversiblen Elemente des Daniell-Typus. Zur praktischen Arbeitsleistung kommt ja nur das Zink-Kupfer-Element in Betracht. In den gewöhnlichen Formen dieses ist der innere Widerstand für Elemente mittlerer Grösse bedeutend, etwa 2 bis $3 \mathrm{Ohm}$. Es ist aber unschwer, dieses zu verbessern, ohne die Grösse des Elementes zu erhöhen. Ich bediene mich seit längerem einer Batterie von Daniell-Elementen folgender Konstruktion: Im porösen Thoncylinder steht ein Cylinder von einer dunnen Bleiplatte geformt mit Durchbohrungen oben und unten far Cirkulation der Flussigkeit. Unten ist der Bleicylinder von einem Netze von Kupferdraht geschlossen, indem er ausser als Elektrode zugleich als Behalter für die Kupfersulfatkrystalle dient. Der Zinkcylinder ausser der Thonzelle ist von einer Lossung von Magnesiumchlorid (2:5) umgeben. Mit einer wirksamen Elektrodenfläche von $400 \mathrm{qcm}$ und einem Abstand zwischen den Elektroden von $2 \mathrm{~cm}$ ist der innere Widerstand nur 0,4 bis $0,5 \mathrm{Ohm}$. Noch geringer wird er, wën man statt Magnesiumchlorid eine Losung von Chlornatrium ( $\mathrm{I}: 3$ ) verwendet; es wird aber dadurch Auskrystallisieren von Natriumsulfat im Thoncylinder veranlasst, wodurch die Haltbarkeit des letzteren noch verringert wird. Sie ist bekanntlich allezeit im Kupfer-ZinkElemente gering; es empfiehlt sich daher, wenn eine Batterie längere Zeit nicht gebraucht wird, den Thoncylinder herauszunehmen und zu entleeren, was bei der oben beschriebenen Konstruktion keine grosse Măhe macht; die Bleielektroden mit den Sulfatkrystallen werden in einer gesăttigten Kupfersulfatlösung hingestellt, da sonst das ausgefallte Kupfer in der Luft bald mit einer Schicht von nichtleitendem basischem Karbonat überzogen wird.

Kopenhagen, November 1898 .

\section{REINIGUNG UND ENTFÄRBUNG \\ ZUCKERHALTIGER FLÜSSIGKEITEN DURCH OZON, DURCH DEN ELEKTRISCHEN STROM UND DURCH VEREINTE ANWENDUNG BEIDER. \\ Von Franz Peters.}

(Mitteilung aus dem elektrochemischen Laboratorium der Technischen Hochschule Berlin.)

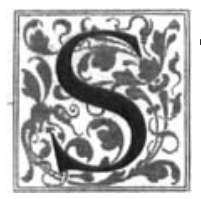

eit ungefahr $184^{1}$ ) hat man sich bemüht, den elektrischen Strom für die Reinigung und Entfarbung von Rubensäten nutzbar zu machen. Von allen seitdem vorgeschlagenen Verfahren ist wohl zuerst das von $H$. Schollmeyer und C. Dammeyer ${ }^{2}$ ) zu technischer Verwendung gelangt. Bei ihm wird der Diffusionssaft zwischen Zink - oder Aluminiumelektroden elektrolysiert. Es entstehen lösliche Metall-Alkaliverbindungen,

I) Englisches Patent Nr. 12335 vom 2I. II. 1848 für W. H. Clement.

e) D. R. P. Nr. 76853 vom .24, Ir. I892. die ausscheidend auf die Verunreinigungen der Säfte, namentlich auf gelöste Eiweissstoffe oder sonstige organische Nichtzuckersubstanzen wirken.

Ozon allein zum Bleichen und Reinigen von Zuckersäften schlug meines Wissens zuerst E. Hagen ${ }^{1}$ ) vor. L. H. Despeissis ${ }^{2}$ ) will durch seine Einwirkung die schon der Elektrolyse unterworfenen Säfte nachbehandeln, wăhrend F a h rig ${ }^{3}$ ) es zur Vorbehandlung in Aussicht nimmt.

I) D. R. P. Nr. 18723 vom 3a. 8. $188 \mathrm{r}$.

2) Englisçhes Patent Nr. $44^{8}$ vom 28. 8. I883

3) Englisches Patent Nr. $355^{6}$ von 1886. 